\title{
Der Einfluss des Sponsoring auf das Markenverhalten von Mitarbeitenden
}
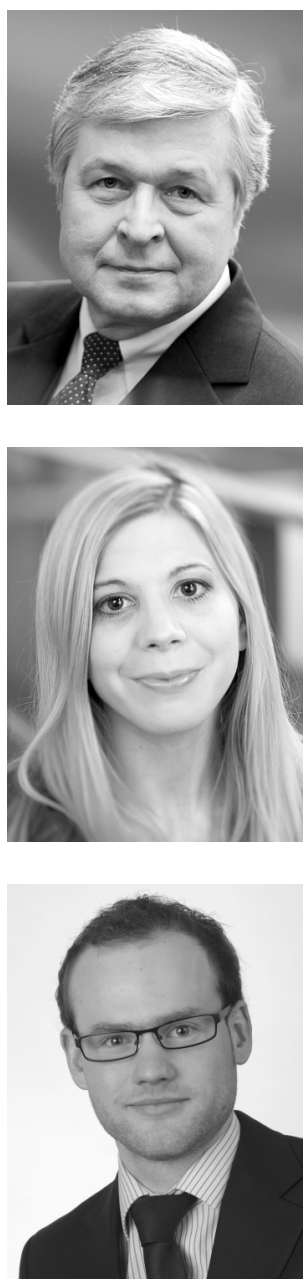

\section{Manfred Brubn, Verena Batt, Matthias Holzer}

Schlüsselbegriffe: Sponsoring, Markenimage, Markencommitment, markenkonformes Mitarbeiterverhalten, Mitarbeiterbefragung

Key Words: Sponsorship, Brand Image, Brand Commitment, Brand Citizenship Behavior, Employee Survey

Zusammenfassung: Mit der Wirkung des Sponsoring auf Mitarbeitende hat sich die wissenschaftliche Literatur bislang kaum auseinandergesetzt, obgleich das Sponsoring als Steuerungsgrösse eines markenkonformen Mitarbeiterverhaltens anzusehen ist. Mit dem vorliegenden Artikel wird dieser Forschungslücke begegnet. Das Ziel ist die Untersuchung der Wirkung des Sponsoring auf das markenbezogene Mitarbeiterverhalten. Hierfür wird ein Modell zur Erfassung der Wirkung des Sponsoring auf markenbezogene Grössen entwickelt. Die Analyse macht deutlich, dass die Sport-, Kultur- und Umweltsponsoringqualität - über die Konstrukte Markenimage und Markencommitment - Einfluss auf das markenkonforme Mitarbeiterverhalten ausübt.

Summary: Research, so far, has insufficiently dealt with the effect of sponsorship on employee behavior, though sponsorship is considered to be a key determinant of brand citizenship behavior. The present article addresses this research gap. The objective is to examine the effect of sponsorship on brand citizenship behavior. Therefore, a model is developed to measure the influence of sponsorship on brand related variables. The analysis reveals that quality of sports, cultural and environmental sponsorship drives - through brand image and brand commitment - brand citizenship behavior.

\section{Relevanz des Sponsoring für die Entstehung eines markenkonformen Mitarbeiterverhaltens}

Das Sponsoring hat sich in den letzten Jahrzehnten zu einem bedeutenden Bestandteil der Kommunikationsstrategie vieler Unternehmen entwickelt. Während 1985 in Deutschland 102 Mio. EUR für Sponsoringaktivitäten aufgewendet wurden (Brubn 2010), lagen die Sponsoringinvestitionen im Jahr 2012 bei 4.4 Mrd. EUR. Für die Jahre 2013 und 2014 wird mit einem weiteren Anstieg auf 4.8 Mrd. EUR gerechnet (FASPO 2013). Unter dem Begriff Sponsoring ist die Analyse, Planung, Umsetzung und Kontrolle sämtlicher Aktivitäten zu verstehen, „die mit der Bereitstellung von Geld, Sachmitteln, Dienstleistungen oder Know-how durch Unternehmen und Institutionen zur Förderung von Personen und/oder 
Organisationen in den Bereichen Sport, Kultur, Soziales, Umwelt und/oder den Medien [...] verbunden sind, um damit gleichzeitig Ziele der Marketing- und Unternehmenskommunikation zu erreichen“ (Brubn 2010, 6f.). Die Begriffsdefinition macht deutlich, dass ein Sponsor seine Fördermittel in der Erwartung, vom Gesponserten eine Gegenleistung zu erhalten, zur Verfügung stellt. Dies steht im Gegensatz zum Mäzenatentum und Spendenwesen, die durch die Förderung von Personen und Institutionen aus altruistischen Motiven heraus gekennzeichnet sind (Hermanns et al. 2007). Eine mögliche Gegenleistung des Gesponserten stellt z.B. die werbewirksame Verwendung des Marken- oder Firmennamens des Sponsors dar (Bruhn 2010).

Die zunehmende Bedeutung in der Unternehmenspraxis legte den Grundstein für eine vertiefte Auseinandersetzung mit dem Sponsoring in der Marketingforschung. Ein zentrales Forschungsfeld stellt in diesem Zusammenhang die Sponsoringwirkungsforschung dar. Hier liegt der Fokus auf der Überprüfung des Erfolges von Sponsoringaktivitäten. In einer Vielzahl von Studien wurde die positive Wirkung des Sponsoring auf markenbezogene Grössen, wie z.B. Markenbekanntheit (Mason/Cochetel 2006; Rowley/Williams 2008), Markenimage (Cornwell et al. 2001; Grohs et al. 2004) oder Markeneinstellung (Huber/ Matthes 2007; Gwinner/Bennett 2008) und darüber auf die Kaufabsicht (Huber/Matthes 2007; Martensen et al. 2007; Mazodier/Merunka 2012), empirisch belegt. Auffallend ist jedoch, dass der Fokus auf den Wirkungen des Sponsoring (extern) bei den Konsumenten liegt. Inwieweit das Sponsoring die Markeneinstellungen und das Markenverhalten von Mitarbeitenden beeinflusst, wurde bislang hingegen noch nicht empirisch untersucht. Neben der externen Wirkung kann das Sponsoring jedoch auch intern, d.h. bei den Mitarbeitenden des als Sponsor tätigen Unternehmens, seine Wirkung entfalten (Brubn 2008). Angesichts des emotionalen Charakters, den das Sponsoring innehat, ist hierbei sogar von besonders positiven Wirkungen auf Mitarbeiterseite auszugehen. So können mit dem Sponsoring Emotionen hervorgerufen werden, die mit anderen Kommunikationsinstrumenten nicht auf vergleichbar authentische Weise zu erzeugen sind (Brubn 2004). Dies wiederum fördert die Entstehung einer emotionalen Beziehung des Mitarbeitenden zur Marke, für die der Mitarbeitende arbeitet (Bruhn 2008); mit der Folge, dass Mitarbeitende ein Verhalten im Sinne der Marke zeigen (Esch/Strödter 2012).

Die Ausführungen machen deutlich, dass das Sponsoring über die Generierung einer emotionalen Bindung des Mitarbeitenden an die Marke als Steuerungsgrösse eines markenkonformen Mitarbeiterverhaltens anzusehen ist. Die Relevanz des Sponsoring gilt umso mehr, als dem markenkonformen Mitarbeiterverhalten eine hohe Bedeutung für den Erfolg der Marke am Markt zugesprochen wird (De Chernatony 2002; Harris 2007; Devasagayam et al. 2010). Verhalten sich Mitarbeitende markenkonform, richten sie - gemäss des identitätsbasierten Markenführungsansatzes - ihr Handeln konsistent an der Markenidentität und am Markenversprechen aus (Burmann/Zeplin 2005). Dadurch machen sie die Markenidentität für die Nachfrager erlebbar und tragen auf diese Weise wesentlich zur Differenzierung der Marke und Präferenzbildung bei den Nachfragern bei (Brexendorfl Tomczak 2004).

Angesichts der Bedeutung, die dem Sponsoring für das Markenverhalten der Mitarbeitenden und damit auch für den Markenerfolg zukommt, besteht die Notwendigkeit einer tiefergehenden Auseinandersetzung mit der Sponsoringwirkung nach innen. Der vorliegende Artikel nimmt sich der hierzu bestehenden Forschungslücke an. Das Ziel ist es, die Wirkung des Sponsoring auf das markenkonforme Mitarbeiterverhalten zu untersuchen. Hier- 
für gilt es, ein Modell zur Erfassung der Wirkungen von Sponsoring auf markenbezogene Grössen zu entwickeln und einer empirischen Überprüfung zu unterziehen.

Der Artikel gliedert sich in fünf Kapitel. Im zweiten Kapitel wird eine Literaturanalyse hinsichtlich der bestehenden Studien zur Wirkung der externen Kommunikation auf Mitarbeitende vorgenommen. Die Literaturaufarbeitung mündet in die Ableitung des Sponsoringwirkungsmodells. Im dritten Kapitel steht die Modellierung der Konstrukte im Mittelpunkt. Des Weiteren werden Hypothesen generiert. Die empirische Überprüfung der hypothetisierten Wirkungszusammenhänge erfolgt im vierten Kapitel. Das fünfte Kapitel bildet das Fazit mit Implikationen für die Unternehmenspraxis und gibt Hinweise für die weiterführende Forschung.

\section{Stand der Forschung zur Wirkung externer Kommunikation auf Mitarbeitende und Ableitung des Sponsoringwirkungsmodells}

Bislang liegt nur eine empirische Studie zur Wirkung des Sponsoring auf Mitarbeitende vor (Hickman et al. 2005). Für die Literaturanalyse wird daher zusätzlich Rückgriff auf Studien genommen, die sich mit der Innenwirkung weiterer externer Kommunikationsinstrumente beschäftigen. Das Ziel der Literaturaufarbeitung ist es, einen Überblick über die bisherigen Studien zur Wirkung externer Kommunikation auf Mitarbeitende zu geben. Darauf basierend wird die Ableitung des hier interessierenden Sponsoringwirkungsmodells vorgenommen.

Tabelle 1 gibt eine Übersicht über die bestehenden Arbeiten. In der Studie zur Sponsoringwirkung wird nachgewiesen, dass eine Affinität des Mitarbeitenden zum vom Unternehmen gesponserten Sportteam in positiver Beziehung zum organisationalen Commitment bzw. zur organisationalen Identifikation des Mitarbeitenden steht (Hickman et al. 2005). Dies unterstreicht die bereits erwähnte Bedeutung des Sponsoring zur Generierung einer emotionalen Bindung von Mitarbeitenden. Die Durchsicht der Studie macht jedoch deutlich, dass der Fokus lediglich auf dem Sportsponsoring liegt. Weitere Erscheinungsformen des Sponsoring, wie z.B. das Kultur-, Sozio- und Umweltsponsoring (Brubn 2010), werden nicht betrachtet. Des Weiteren werden mit dem Commitment und der Identifikation lediglich psychologische Grössen betrachtet. Inwieweit das Sponsoring über diese Konstrukte Einfluss auf das Mitarbeiterverhalten nimmt, findet in der empirischen Untersuchung keine Berücksichtigung. Schliesslich ist auf den fehlenden Markenbezug hinzuweisen. In der Studie von Hickman et al. (2005) interessiert die Wirkung des Sponsoring auf die emotionale Bindung des Mitarbeitenden an das Unternehmen. Dies steht im Gegensatz zur vorliegenden Studie, die sich der Sponsoringwirkung auf markenbezogene Grössen widmet.

Mit Ausnahme der Studie zur Sponsoringwirkung fokussieren sich die weiteren Arbeiten auf die Innenwirkung der Werbung. Weitere Kommunikationsinstrumente erfahren keine Berücksichtigung. In den Studien wird dabei mehrheitlich die Ausgestaltung der Werbung und deren Einfluss auf die Mitarbeitenden untersucht. Hinsichtlich der Ausgestaltung der Werbung wird zum einen der von den Mitarbeitenden wahrgenommene Übertreibungsgrad der Werbung im Hinblick auf das dargestellte Mitarbeiterverhalten betrachtet (Wolfinbarger/Gilly 2005; Wentzel et al. 2009, 2010). Zum anderen liegt der Fokus auf der aus Mitarbeitersicht korrekten Darstellung der Mitarbeitenden, des Werbeversprechens, der Organisation in der Werbung, auf der wahrgenommenen Wirksamkeit der Werbung sowie der wahrgenommenen Kongruenz zwischen den persönlichen Werten des Mitarbeitenden 
und den in der Werbung vermittelten Werten (Gilly/Wolfinbarger 1998; Wolfinbarger/ Gilly 2005; Wolfinbarger Celsi/Gilly 2010).

Von diesen Arbeiten grenzen sich Hughes (2013) und Acito (1980) ab. Die Autoren widmen sich weniger der konkreten Ausgestaltung der Werbung, als vielmehr der allgemein wahrgenommenen Quantität sowie Qualität bzw. Einstellung gegenüber der Werbung. Die Quantität der Werbung spiegelt die vom Mitarbeitenden wahrgenommene Häufigkeit der Werbung wider. Die Qualität der Werbung stellt die mitarbeiterseitige Bewertung der Werbung dar. Hinsichtlich der Wirkung der Werbewahrnehmung zeigen die Studien, dass eine als ansprechend wahrgenommene Werbegestaltung (z.B. eine korrekte Darstellung der Mitarbeitenden) und eine hohe wahrgenommene Quantität und Qualität der Werbung positive Wirkungen beim Mitarbeitenden hervorruft. Die primär untersuchten Wirkungsgrössen stellen die organisationale Identifikation, das organisationale Commitment und der organisationale Stolz dar. Verhaltensbezogene Grössen werden nur vereinzelt betrachtet (z.B. Markenanstrengungen bei Hughes 2013, Kundenorientierung bei Wolfinbarger Celsi/Gilly 2010). Des Weiteren wird deutlich, dass in den Arbeiten, mit Ausnahme der Studie von Hughes (2013), kein Markenbezug vorliegt. Ebenso wie bei der Studie von Hickman et al. (2005) liegt deren Fokus vielmehr auf dem Einfluss der Werbung auf organisationsbezogene Grössen.

\begin{tabular}{|c|c|c|c|c|}
\hline Autor (Jahr) & $\begin{array}{c}\text { Stichprobe/ } \\
\text { Analysemethode }\end{array}$ & $\begin{array}{c}\text { Unabhängige } \\
\text { Variable(n) }\end{array}$ & $\begin{array}{l}\text { Abhängige } \\
\text { Variable(n) }\end{array}$ & Zentrale Ergebnisse \\
\hline $\begin{array}{l}\text { Acito } \\
(1980)\end{array}$ & $\begin{array}{l}\mathrm{n}=342 \text { Mitarbei- } \\
\text { tende einer Bank; } \\
\text { Regressionsanalyse }\end{array}$ & $\begin{array}{l}\text { - Einstellung } \\
\text { gegenüber der } \\
\text { Werbung }\end{array}$ & $\begin{array}{l}\text { - Arbeitszufrie- } \\
\text { denheit } \\
\text { - Job Invovement } \\
\text { - Ausmass Kun- } \\
\text { denkontakte } \\
\text { - Ausmass Kon- } \\
\text { takte mit ande- } \\
\text { ren Mitarbei- } \\
\text { tenden }\end{array}$ & $\begin{array}{l}\text { Die Einstellung der Mitarbei- } \\
\text { tenden gegenüber der Wer- } \\
\text { bung wirkt positiv auf sämtli- } \\
\text { che abhängige Variablen. }\end{array}$ \\
\hline $\begin{array}{l}\text { Gilly/ } \\
\text { Wolfinbarger } \\
\text { (1998) }\end{array}$ & $\begin{array}{l}\mathrm{n}=151 \text { Mitarbei- } \\
\text { tende aus Dienst- } \\
\text { leistungs- und In- } \\
\text { dustriegüterunter- } \\
\text { nehmen; Inter- } \\
\text { views }\end{array}$ & $\begin{array}{l}\text { Wertekongru- } \\
\text { enz der Wer- } \\
\text { bung } \\
\text { - Korrektheit } \\
\text { der Werbung } \\
\text { - Wirksamkeit } \\
\text { der Werbung }\end{array}$ & $\begin{array}{l}\text { - Rollenkonflikt } \\
\text { - Organisationa- } \\
\text { ler Stolz } \\
\text { - Organisationale } \\
\text { Identifikation } \\
\text { - Organisationa- } \\
\text { les Commit- } \\
\text { ment }\end{array}$ & $\begin{array}{l}\text { - Die wahrgenommene Wertein- } \\
\text { kongruenz der Werbung be- } \\
\text { einflusst Identifikation und } \\
\text { Commitment negativ. } \\
\text { - Die wahrgenommene Unkor- } \\
\text { rektheit der Werbung beein- } \\
\text { flusst Stolz, Identifikation und } \\
\text { Commitment negativ, den } \\
\text { Rollenkonflikt positiv. } \\
\text { - Die wahrgenommene Wirk- } \\
\text { samkeit der Werbung beein- } \\
\text { flusst Identifikation und Com- } \\
\text { mitment positiv. }\end{array}$ \\
\hline
\end{tabular}




\begin{tabular}{|c|c|c|c|c|}
\hline Autor (Jahr) & $\begin{array}{c}\text { Stichprobe/ } \\
\text { Analysemethode }\end{array}$ & $\begin{array}{c}\text { Unabhängige } \\
\text { Variable(n) }\end{array}$ & $\begin{array}{l}\text { Abhängige } \\
\text { Variable(n) }\end{array}$ & Zentrale Ergebnisse \\
\hline $\begin{array}{l}\text { Wolfinbar- } \\
\text { ger/Gilly } \\
(2005)\end{array}$ & $\begin{array}{l}\text { Studie 1: } \mathrm{n}=607 \\
\text { Mitarbeitende ei- } \\
\text { nes Technologie- } \\
\text { unternehmens } \\
\text { Studie 2: } \mathrm{n}=472 \\
\text { Mitarbeitende ei- } \\
\text { nes Krankenhau- } \\
\text { ses; } \\
\text { Strukturglei- } \\
\text { chungsanalyse }\end{array}$ & $\begin{array}{ll}\text { - } & \text { Korrekte Dar- } \\
\text { stellung der } \\
\text { Organisation } \\
\text { - } \\
\text { Korrekte Dar- } \\
\text { stellung der } \\
\text { Mitarbeiten- } \\
\text { den } \\
\text { - Wirksamkeit } \\
\text { der Werbung } \\
\text { - Übertrei- } \\
\text { bungsgrad } \\
\text { der Werbung }\end{array}$ & $\begin{array}{l}\text { Einstellung ge- } \\
\text { genüber der } \\
\text { Organisation }\end{array}$ & $\begin{array}{l}\text { - Die korrekte Darstellung der } \\
\text { Organisation bzw. der Mitar- } \\
\text { beitenden in der Werbung } \\
\text { wirkt positiv auf die Einstel- } \\
\text { lung. } \\
\text { - Die wahrgenommene Wirk- } \\
\text { samkeit der Werbung wirkt } \\
\text { positiv auf die Einstellung. } \\
\text { - Der Übertreibungsgrad der } \\
\text { Werbung wirkt negativ auf } \\
\text { die Einstellung. }\end{array}$ \\
\hline $\begin{array}{l}\text { Hickman et } \\
\text { al. } \\
\text { (2005) }\end{array}$ & $\begin{array}{l}\mathrm{n}=502 \text { Mitarbei- } \\
\text { tende; } \\
\text { Korrelations- } \\
\text { analyse }\end{array}$ & $\begin{array}{l}\text { Affinität ge- } \\
\text { genüber } \\
\text { Sponsoring- } \\
\text { objekt }\end{array}$ & $\begin{array}{l}\text { - Organisationa- } \\
\text { les Commit- } \\
\text { ment } \\
\text { - Organisationale } \\
\text { Identifikation }\end{array}$ & $\begin{array}{l}\text { Die Affinität gegenüber dem } \\
\text { Sponsoringobjekt steht in po- } \\
\text { sitiver Beziehung zu Commit- } \\
\text { ment und Identifikation. }\end{array}$ \\
\hline $\begin{array}{l}\text { Wentzel et al. } \\
(2009,2010)\end{array}$ & $\begin{array}{l}\text { 2009: } \\
\text { Studie 1: } \mathrm{n}=101 \\
\text { Studie 2: } \mathrm{n}=102 \\
\text { 2010: } \\
\text { Studie 1: } \mathrm{n}=102 \\
\text { Studie 2: } \mathrm{n}=122 \\
\text { jeweils Mitarbei- } \\
\text { tende einer Bank; } \\
\text { Experimentelle } \\
\text { Untersuchung }\end{array}$ & $\begin{array}{l}\text { Übertrei- } \\
\text { bungsgrad } \\
\text { des Mitarbei- } \\
\text { terverhaltens } \\
\text { in der Wer- } \\
\text { bung }\end{array}$ & $\begin{array}{l}\text { Motivation zur } \\
\text { Steigerung der } \\
\text { eigenen Leis- } \\
\text { tung }\end{array}$ & $\begin{array}{l}\text { - Leicht übertreibende Werbung } \\
\text { erhöht die Motivation zur } \\
\text { Steigerung der eigenen Leis- } \\
\text { tung, während stark übertrei- } \\
\text { bende Werbung die Motivati- } \\
\text { on senkt. } \\
\text { - Dies gilt jedoch nur im Falle } \\
\text { einer hohen Ähnlichkeit zwi- } \\
\text { schen Mitarbeitendem und } \\
\text { Vorbild in der Werbung bzw. } \\
\text { dann, wenn der Mitarbeitende } \\
\text { ein Entity-Theoretiker ist. }\end{array}$ \\
\hline $\begin{array}{l}\text { Wolfinbarger } \\
\text { Celsi/Gilly } \\
(2010)\end{array}$ & $\begin{array}{l}\text { Studie } 1: \mathrm{n}=607 \\
\text { Mitarbeitende ei- } \\
\text { nes Technologie- } \\
\text { unternehmens } \\
\text { Studie 2: } \mathrm{n}=472 \\
\text { Mitarbeitende ei- } \\
\text { nes Krankenhau- } \\
\text { ses; } \\
\text { Strukturglei- } \\
\text { chungsanalyse }\end{array}$ & 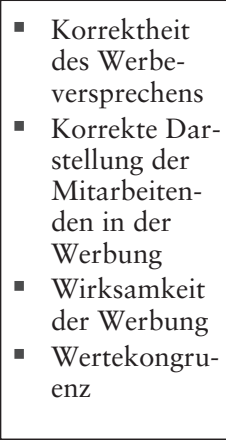 & $\begin{array}{l}\text { - Organisationa- } \\
\text { ler Stolz } \\
\text { - Kundenorien- } \\
\text { tierung }\end{array}$ & $\begin{array}{l}\text { - Die Korrektheit des Werbe- } \\
\text { versprechens und die korrekte } \\
\text { Darstellung der Mitarbeiten- } \\
\text { den in der Werbung sowie die } \\
\text { Wertekongruenz wirken posi- } \\
\text { tiv auf die wahrgenommene } \\
\text { Wirksamkeit der Werbung. } \\
\text { - Die wahrgenommene Wirk- } \\
\text { samkeit der Werbung wirkt } \\
\text { positiv auf den Stolz. } \\
\text { - Der Stolz von Mitarbeitenden } \\
\text { wirkt positiv auf die Kunden- } \\
\text { orientierung. }\end{array}$ \\
\hline $\begin{array}{l}\text { Hughes } \\
\text { (2013) }\end{array}$ & $\begin{array}{l}\mathrm{n}=197 \text { Vertriebs- } \\
\text { mitarbeitende; } \\
\text { Strukturglei- } \\
\text { chungsanalyse }\end{array}$ & $\begin{array}{l}\text { - Quantität der } \\
\text { Werbung } \\
\text { - Qualität der } \\
\text { Werbung }\end{array}$ & $\begin{array}{l}\text { - Markenidentifi- } \\
\text { kation } \\
\text { - Ergebniserwar- } \\
\text { tung } \\
\text { - Markenan- } \\
\text { strengungen }\end{array}$ & $\begin{array}{l}\text { - Die wahrgenommene Quanti- } \\
\text { tät der Werbung wirkt positiv } \\
\text { auf die Ergebniserwartung. } \\
\text { - Die wahrgenommene Qualität } \\
\text { der Werbung wirkt positiv auf } \\
\text { die Markenidentifikation. } \\
\text { - Die Markenidentifikation und } \\
\text { die Ergebniserwartung wirken } \\
\text { positiv auf die Markenan- } \\
\text { strengungen. }\end{array}$ \\
\hline
\end{tabular}

Tabelle 1: Studien zur Wirkung externer Kommunikation auf Mitarbeitende 
Die Literaturanalyse macht deutlich, dass die externe Kommunikation, neben der Wirkung auf Konsumenten, auch Einfluss auf die Mitarbeitenden des kommunikationsführenden Unternehmens ausübt. Aufbauend auf der Literaturbestandsaufnahme folgt an dieser Stelle die Ableitung des hier interessierenden Sponsoringwirkungsmodells. Wie bereits erwähnt, wurde bei Hickman et al. (2005) lediglich das Sportsponsoring berücksichtigt. In der vorliegenden Studie soll eine umfassendere Analyse der Innenwirkung des Sponsoring auf Mitarbeitende stattfinden, indem neben dem Sportsponsoring auch die weiteren Erscheinungsformen des Sponsoring berücksichtigt werden. Diese sind das Kultur-, Sozio- und Umweltsponsoring. In Anlehnung an Hughes (2013) liegt der Fokus auf der mitarbeiterseitig wahrgenommenen Qualität der Sponsoringarten. Dadurch wird eine globale Betrachtung der Sponsoringwahrnehmung durch die Mitarbeitenden ermöglicht. Als direkte Wirkungsgrösse der Sponsoringqualität wird das Markenimage, das hier als Einstellung des Mitarbeitenden gegenüber der Marke verstanden wird, betrachtet. Dieses Vorgehen wird damit begründet, dass zunächst eine positive Haltung gegenüber der Marke vorzuliegen hat, damit eine emotionale Bindung des Mitarbeitenden an die Marke, d.h. Markencommitment, entstehen kann. Das Markencommitment wird im Modell als direkte Determinante des markenkonformen Mitarbeiterverhaltens abgebildet. In Abbildung 1 ist das entwickelte Untersuchungsmodell grafisch dargestellt. Nachfolgend liegt der Fokus auf der Konzeptualisierung der Konstrukte und Ableitung von Hypothesen.

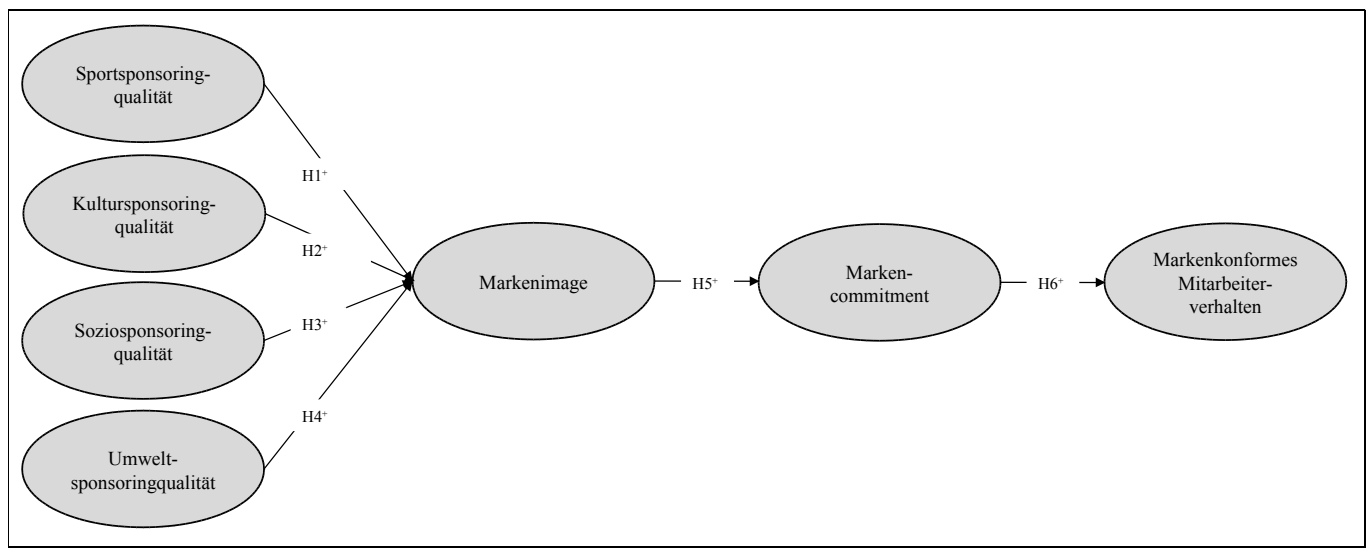

Abbildung 1: Untersuchungsmodell der Sponsoringwirkung

\section{Konzeptualisierung der Konstrukte und Hypothetisierung}

\subsection{Sponsoringqualität und Markenimage}

Unter Sponsoringqualität wird, in Anlehnung an Hughes (2013), die Bewertung des Sponsoring durch Mitarbeitende verstanden. Das Konstrukt zielt folglich auf die Art und Weise ab, wie Sponsoringengagements durch Mitarbeitende wahrgenommen werden, sowie die Erwartungen, die Mitarbeitende an Sponsoringengagements haben. Wie bereits in Kapitel 2 erwähnt, wird im Rahmen dieser Studie die Sport-, Kultur-, Sozio- und Umweltsponsoringqualität untersucht.

Das Markenimage wird nach Keller (1993) als die Summe aller perzipierten und im Gedächtnis gespeicherten Assoziationen, die ein Individuum mit einer Marke verbindet, defi- 
niert. Starke, positive und einzigartige Markenassoziationen führen zu einem überlegenen Markenimage. Assoziationen lassen sich durch Eigenschaften, Nutzen und Einstellungen charakterisieren. In der vorliegenden Studie liegt der Schwerpunkt auf den Einstellungen der Mitarbeitenden gegenüber der Marke. Diese werden über kognitive und affektive Merkmale erfasst (Batra/Stephens 1994; Lardinoit/Quester 2001; Madden et al. 1988).

Im Bereich der Attitude Toward the Ad-Forschung belegt eine Reihe von Studien die Wichtigkeit der Einstellung von Konsumenten gegenüber der Werbung als wesentlicher Indikator für die positive Einstellung von Konsumenten gegenüber der werbenden Marke (Bongard 2002). In diesem Zusammenhang stellten Burke und Edell (1989) fest, dass eine positive Einstellung des Konsumenten gegenüber der Werbung die Beurteilung der werbenden Marke positiv beeinflusst und umgekehrt eine negative Einstellung einen negativen Einfluss hat.

Überträgt man diese Erkenntnis analog auf das Sponsoring, so kann die Einstellung gegenüber der Werbemassnahme mit der Einstellung der Mitarbeitenden gegenüber dem Sponsoringengagement verglichen werden. Die Einstellung gegenüber dem Sponsoringengagement wird in der vorliegenden Studie als Sponsoringqualität erfasst. Folglich ist anzunehmen, dass eine positive Wahrnehmung der Sponsoringqualität die Beurteilung des Markenimages durch die Mitarbeitenden positiv beeinflusst.

Die obigen Erkenntnisse führen zu folgenden Hypothesen der Sponsoringqualität:

H1: Das Markenimage wird von der Sportsponsoringqualität positiv beeinflusst.

H2: Das Markenimage wird von der Kultursponsoringqualität positiv beeinflusst.

H3: Das Markenimage wird von der Soziosponsoringqualität positiv beeinflusst.

H4: Das Markenimage wird von der Umweltsponsoringqualität positiv beeinflusst.

\subsection{Markencommitment}

Unter Markencommitment wird die emotionale, d.h. gefühlsmässige, Bindung des Mitarbeitenden zur Marke verstanden (O’Reilly III/Chatman 1986; Zeplin 2006; Maloney 2007; Giersch 2008; König 2010; Piehler 2011; Batt 2013). Ein hohes Markencommitment äussert sich darin, dass sich die Mitarbeitenden der Marke verbunden fühlen und stolz darauf sind, Mitarbeitende der Marke zu sein. Gemäss der Theorie der sozialen Identität verwenden Individuen die Zugehörigkeit zu sozialen Gruppen, um ihre eigene Persönlichkeit zu definieren. Unternehmen stellen eine solche soziale Gruppe dar. Als Konsequenz hieraus versuchen Individuen insbesondere solchen Unternehmen anzugehören, die mit positiven Eigenschaften assoziiert werden und einen guten Ruf bzw. ein gutes Markenimage geniessen (Brammer et al. 2007; Panagopoulos et al. 2011). Haben Individuen das Ziel erreicht, für ein Unternehmen arbeiten zu können, das aus ihrer Sicht ein gutes Markenimage aufweist, findet durch die Zugehörigkeit zu dieser sozialen Gruppe eine Internalisierung der Markenidentität durch die Mitarbeitenden statt. Mithin wird eine positive Wirkung auf den Stolz und die Verbundenheit der Mitarbeitenden zum Unternehmen und damit auf deren Markencommitment bewirkt. Folgende Hypothese wird abgeleitet:

H5: Das Markencommitment wird durch das Markenimage positiv beeinflusst. 


\subsection{Markenkonformes Mitarbeiterverhalten}

Das markenkonforme Mitarbeiterverhalten entspricht inhaltlich dem in der Literatur häufig verwendeten Konstrukt Brand Citizenship Behavior (BCB) (Zeplin 2006; Maloney 2007; König 2010; Piehler 2011). Darunter werden sämtliche Verhaltensweisen eines Mitarbeitenden subsumiert, die konform zur Markenidentität respektive zum Markennutzenversprechen sind und im Ergebnis die Markenidentität stärken (Piehler 2011). In Anlehnung an die Konzeptualisierung des BCB nach Maloney (2007) wird für die vorliegende Studie das von Batt (2013) entwickelte markenkonforme Mitarbeiterverhalten als dreidimensionales Konstrukt übernommen.

Die Dimension Markenakzeptanz umfasst alle Verhaltensweisen, die von einem Mitarbeitenden als Repräsentant der Marke erwartet werden, wie beispielsweise das Einhalten des Markenversprechens im Kundenkontakt (Tomczak et al. 2008). Die Dimensionen Markenmissionierung und Markenpartizipation beziehen sich auf Verhaltensweisen, die freiwillig und ausserhalb von formalisierten Rollenerwartungen gezeigt werden. Die Dimension Markenmissionierung ist nach aussen, d.h. an die externen Zielgruppen, gerichtet und bezieht sich auf das bewusste Einsetzen des Mitarbeitenden für die Belange der Marke (z.B. die Weiterempfehlung oder Verteidigung der Marke). Markenpartizipation ist nach innen, d.h. gegenüber den internen Zielgruppen bzw. der markenführenden Institution, gerichtet und beinhaltet Verhaltensweisen, die darauf abzielen, aktiven Einfluss auf die Weiterentwicklung der Markenidentität zu nehmen und sich selbst im Sinne der Marke weiterzubilden (z.B. durch das Lesen von markenbezogenem Informationsmaterial oder den Besuch markenbezogener Schulungen) (Maloney 2007; Batt 2013).

Auf Basis der Theorie der sozialen Identität lässt sich ein positives Ursache-WirkungsVerhältnis zwischen dem Markencommitment und markenkonformen Mitarbeiterverhalten ableiten. Mitarbeitende, die ein hohes Commitment zur Marke aufweisen, identifizieren sich mit dieser und leiten aus der Beziehung zu ihr die eigene soziale Identität ab. Eine Marke, die wenig Erfolg am Markt hat, tritt dem Streben des Mitarbeitenden nach einer positiven sozialen Identität entgegen. Mitarbeitende, die ein hohes Commitment zur Marke aufweisen, engagieren sich daher für diese, um sie zu unterstützen (z.B. hinsichtlich ihres Erfolgs am Markt), um letzten Endes ihre eigene, aus der Identifikation mit der Marke abgeleitete, soziale Identität zu schützen (vgl. ähnlich Aub et al. 2007; Bryant 2010; Batt 2013). Folgende Hypothese wird daraus abgeleitet:

H6: Das markenkonforme Mitarbeiterverhalten wird durch das Markencommitment positiv beeinflusst.

\section{Ergebnisse der empirischen Untersuchung}

\subsection{Design und Durchführung der empirischen Untersuchung}

Die Überprüfung des Hypothesensystems erfolgte auf Basis einer empirischen Untersuchung. Die hierfür erforderlichen Daten wurden im Rahmen einer anonymen schriftlichen Mitarbeiterbefragung in einem schweizerischen Unternehmen erhoben. ${ }^{1}$ Das Unternehmen setzt ein umfangreiches Sponsoringengagement ein, weshalb es zur Überprüfung des Hy-

1 Auf Wunsch des Unternehmens wird auf die Nennung des Unternehmens sowie der Branche, in der das Unternehmen tätig ist, verzichtet. 
pothesensystems als geeignet angesehen wird. Zur besseren Erreichbarkeit der Mitarbeitenden wurde die Befragung online durchgeführt.

Der Link zum Fragebogen wurde nach dem Zufallsprinzip an 600 Mitarbeitende in der Deutschschweiz verschickt. 236 vollständig ausgefüllte Fragebogen wurden generiert. Dies entspricht einer Rücklaufquote von 39.3 Prozent. Die Stichprobe setzt sich aus 61 Prozent männlichen und 39 Prozent weiblichen Probanden zusammen. Die Altersstruktur zeigt sich wie folgt: 8.9 Prozent der Probanden sind jünger als 26 Jahre, 54.2 Prozent der Probanden sind zwischen 26 und 45 Jahre alt und 36.9 Prozent der Probanden sind älter als 46 Jahre alt. 45.3 Prozent der Probanden sind seit mehr als zehn Jahren, 18.2 Prozent der Probanden sind seit sechs bis zehn Jahren, 20.3 Prozent sind seit drei bis fünf Jahren, 8.1 Prozent sind seit ein bis zwei Jahren und 8.1 Prozent sind seit weniger als einem Jahr für das Unternehmen tätig. 60.6 Prozent der Probanden führen eine Bürotätigkeit aus, 32.7 Prozent üben eine leitende Position aus, 5.1 Prozent sind in der Produktion tätig, jeweils 0.8 Prozent sind im Verkauf und in der Geschäftsführung angestellt. 40.7 Prozent der Probanden haben Kundenkontakt.

\subsection{Operationalisierung und Validierung der Konstrukte}

Die Messung der Modellkonstrukte erfolgte auf einer 7-stufigen Likertskala mit den Ex-

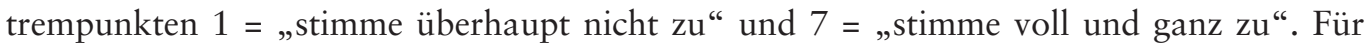
sämtliche Grössen wurde eine reflektive Operationalisierung gewählt. Dieser Entscheidung liegt die Überlegung zugrunde, dass die einzelnen Indikatoren die Konstrukte widerspiegeln. Die Indikatoren sind also nicht unabhängig voneinander bzw. die Veränderung eines Indikators bewirkt die Veränderung aller Indikatoren (Jarvis et al. 2003). Tabelle A1 (Anhang) gibt einen Überblick über die Messitems. Es wurde auf in der Literatur bewährte Skalen Rückgriff genommen. Bei den Konstrukten Sportsponsoringqualität, Kultursponsoringqualität, Soziosponsoringqualität, Umweltsponsoringqualität, Markenimage und markenkonformes Mitarbeiterverhalten erfolgte eine Anpassung an die Gegebenheiten der vorliegenden Studie. Hierbei fand die Tatsache, dass mit Sport-, Kultur-, Sozio- und Umweltsponsoring vier unterschiedliche Sponsoringarten untersucht werden, besondere Berücksichtigung. Mit Ausnahme des Konstrukts markenkonformes Mitarbeiterverhalten wurden sämtliche Konstrukte eindimensional gemessen. Die Operationalisierung des markenkonformen Mitarbeiterverhaltens erfolgte als Konstrukt zweiter Ordnung über drei Dimensionen (Markenakzeptanz, Markenmissionierung und Markenpartizipation). Hierzu wurden Dimensionswerte (Indizes) berechnet. Für die Messitems des Konstrukts markenkonformes Mitarbeiterverhalten vgl. Batt (2013).

Die Messmodelle wurden mit Hilfe von SPSS 21 und Amos 7.0 als Maximum Likelihood-Schätzung analysiert. In Tabelle A1 (Anhang) sind die Ergebnisse dargestellt. Um die Güte der Messmodelle zu überprüfen, erfolgte die Integration aller abhängigen und unabhängigen Konstrukte in ein multifaktorielles Modell (Homburg/Jensen 2007). Die Ergebnisse bescheinigen den Modellen einen guten Modellfit. Des Weiteren weisen sämtliche Konstrukte signifikante Faktorladungen auf. Die erklärte Varianz liegt bei jedem Konstrukt über dem geforderten Wert von 50 Prozent (Homburg/Giering 1996). Das $\alpha$ (Cronbach'sches Alpha) verweist mit Werten von über 0.7 auf ein hohes Mass an Reliabilität und Konvergenzvalidität der Konstrukte. Mittels des Composite-Reliability-Werts wurde die Konstruktreliabilität gemessen. Alle Konstrukte genügen dem geforderten Mindestwert 
von 0.7. Die durchschnittlich erfasste Varianz (DEV) der untersuchten Konstrukte liegt über dem Grenzwert von 0.5.

Tabelle A2 (Anhang) zeigt die Ergebnisse der Überprüfung der Diskriminanzvalidität anhand des Fornell Larcker-Kriteriums (Fornell/Larcker 1981). Mit Ausnahme der Konstrukte Markencommitment und markenkonformes Mitarbeiterverhalten sind die Wurzeln der DEV-Werte stets grösser als die Werte der Faktorkorrelationen. Dies lässt auf Diskriminanzvalidität schliessen. Für die Konstrukte Markencommitment und markenkonformes Mitarbeiterverhalten wurde zusätzlich ein Х2-Differenztest durchgeführt. Der Wert lag über den geforderten 3.84 (Homburg 1998). Folglich wird auch hinsichtlich dieser Konstrukte auf Diskriminanzvalidität geschlossen.

\subsection{Schätzung des Kausalmodells und Modellbeurteilung}

Das hypothetisierte Wirkungsmodell wurde mittels Strukturgleichungsanalyse (Maximum Likelihood-Schätzung) empirisch überprüft. Die in Abbildung 2 vorgestellten Ergebnisse zeigen, dass das Wirkungsmodell die Empirie gut abbildet. Das Bestimmtheitsmass $\mathrm{R}^{2}$ weist für alle endogenen Konstrukte aussagekräftige Werte auf. Des Weiteren offenbart die Modellprüfung signifikante Zusammenhänge zwischen den Konstrukten. Einzig der Wirkungspfad zwischen Soziosponsoringqualität und Markenimage ist nicht signifikant. Die Hypothesen H1, H3, H4, H5 und H6 sind somit anzunehmen, wohingegen Hypothese $\mathrm{H} 2$ abzulehnen ist.

\section{Fazit, Implikationen und Ausblick}

Ausgangspunkt dieses Artikels war die Erkenntnis, dass das Sponsoring als Steuerungsgrösse eines markenkonformen Mitarbeiterverhaltens anzusehen ist. Bisher mangelt es aber an Untersuchungen, die sich mit der Innenwirkung des Sponsoring beschäftigen. Die vorliegende Studie setzt an dieser Forschungslücke an. Das Ziel lag in der Untersuchung der Wirkung des Sponsoring auf das markenbezogene Mitarbeiterverhalten. Hierfür wurde ein Modell zur Erfassung der Wirkung des Sponsoring auf markenbezogene Grössen entwickelt und einer empirischen Überprüfung unterzogen.

Die Ergebnisse der Modellprüfung unterstreichen die Relevanz des Sponsoring für die Entstehung eines markenkonformen Mitarbeiterverhaltens. So zeigt die Analyse, dass die Konstrukte Sport-, Kultur- und Umweltsponsoringqualität auf das Markenimage wirken und darüber auf das Markencommitment und das markenkonforme Mitarbeiterverhalten. Des Weiteren wird die Bedeutung des Sponsoring als imagebildendes Kommunikationsinstrument deutlich. So wird mehr als die Hälfte der Varianz des Markenimages von Mitarbeitenden durch die Sponsoringkonstrukte erklärt. Den stärksten Einfluss auf das Markenimage, und damit indirekt auch auf das markenkonforme Mitarbeiterverhalten, übt die Kultursponsoringqualität aus, gefolgt von der Sportsponsoringqualität und der Umweltsponsoringqualität. Der Zusammenhang zwischen der Soziosponsoringqualität und dem Markenimage findet hingegen keinen empirischen Beleg. Kultur-, Sport- und Umweltsponsoringmassnahmen beziehen sich auf Events, die gemeinsam mit anderen Person erlebt werden können (z.B. Sportturniere, Messen). Das Soziosponsoring des betrachteten Unternehmens legt hingegen das Augenmerk auf die Unterstützung sozialer Einrichtungen. Die mit Sponsoringmassnahmen verbundenen Erlebnisse fördern die Herausbildung des Mar- 


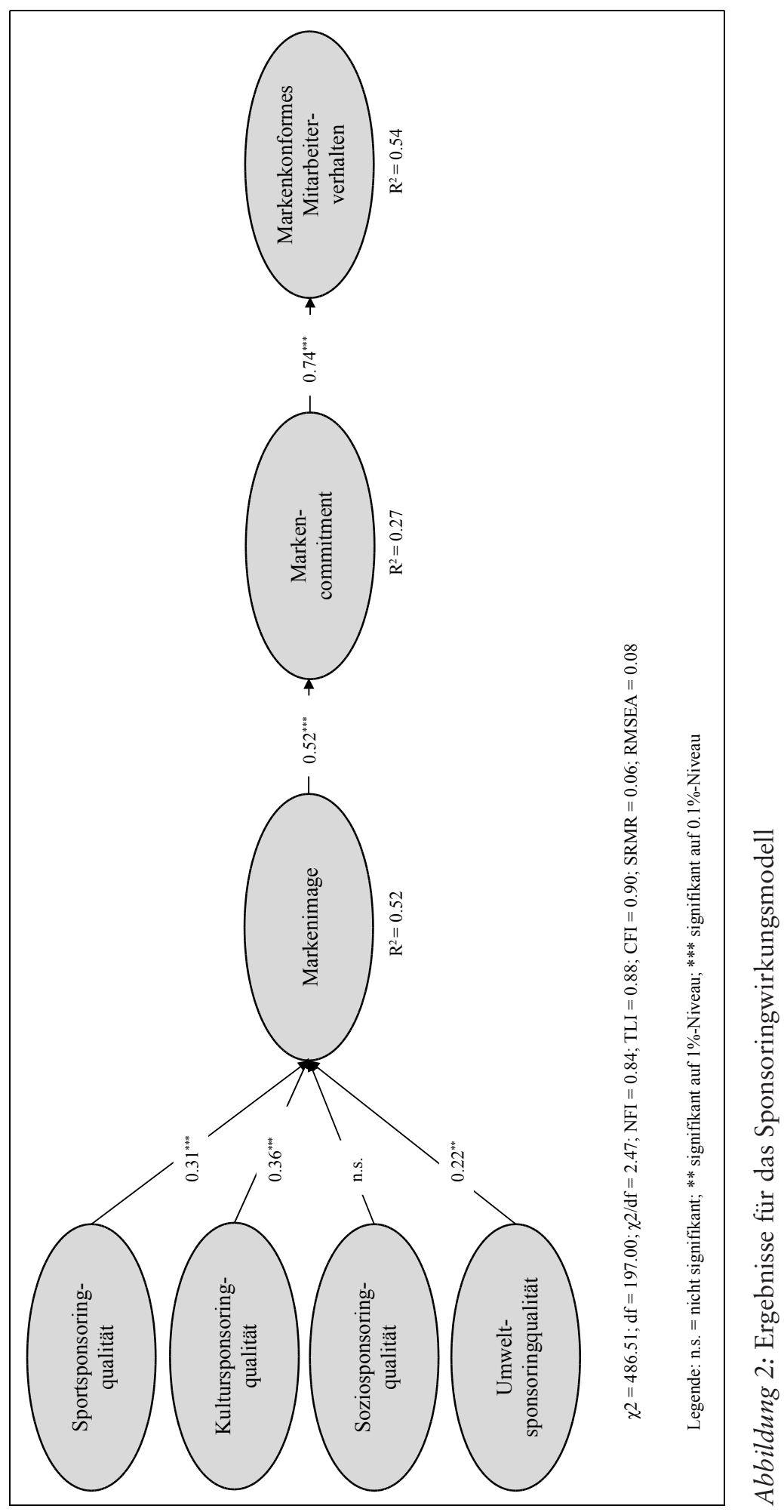


kenimages. Möglicherweise ist die fehlende Signifikanz somit auf den vergleichsweise geringen Erlebnischarakter der Soziosponsoringmassnahmen zurückzuführen.

Aus den Ergebnissen lassen sich folgende Implikationen für die Unternehmenspraxis ableiten: Für die Steuerung eines markenkonformen Mitarbeiterverhaltens durch das Sponsoring haben Unternehmen darauf zu achten, dass die Sponsoringaktivitäten von den Mitarbeitenden positiv wahrgenommen werden. Hierfür eignet sich ein regelmässiges Monitoring hinsichtlich der mitarbeiterseitigen Wahrnehmung des Sponsoring, um bei Bedarf entsprechende Modifikationen in der Ausgestaltung des Sponsoring in die Wege zu leiten. Des Weiteren sind im Rahmen von Mitarbeiterbefragungen die Erwartungen von Mitarbeitenden an das Sponsoring zu identifizieren. Diese Erwartungen sind bei der späteren Planung und Umsetzung der Sponsoringaktivitäten zu berücksichtigen. Es ist anzunehmen, dass sich ein Sponsoringobjekt (z.B. ein Fussballverein), das die Interessen der Mitarbeitenden widerspiegelt, positiv auf die Beurteilung des Sponsoring auswirkt. Daher empfiehlt es sich, bei der Auswahl des Sponsoringobjekts, neben den Interessen der Kunden, auch die der Mitarbeitenden zu berücksichtigen. Dies gilt insbesondere für Dienstleistungsunternehmen, bei denen das Internal Branding eine herausragende Bedeutung aufweist und bei denen das eigen- und fremdinitiierte Sponsoring in ihren verschiedenen Varianten eine besondere Rolle spielt.

Der vorliegende Artikel unterliegt auch Limitationen. Daraus ergibt sich in folgender Hinsicht Forschungsbedarf. Die empirische Untersuchung wurde lediglich in einem einzelnen Unternehmen durchgeführt. Somit ist denkbar, dass die Ergebnisse von den durchgeführten Sponsoringaktivitäten des betrachteten Unternehmens abhängig sind. Dies gilt insbesondere für den nicht signifikanten Wirkungszusammenhang zwischen der Soziosponsoringqualität und dem Markenimage. Forschungsbedarf besteht demzufolge hinsichtlich einer Replikation der Studie in weiteren Unternehmen. Des Weiteren ist zu überprüfen, inwieweit die Zusammenhänge zwischen den Konstrukten von bestimmten Kriterien, wie z.B. (Dienst-)Alter, Bildungsgrad, Markeninvolvement, Arbeitsbereich oder persönlichen Interessen, abhängig sind. Es ist z.B. zu vermuten, dass bei sportinteressierten Mitarbeitenden der Zusammenhang zwischen der Sportsponsoringqualität und dem Markenimage höher ist als bei weniger sportaffinen Beschäftigten. Studien zur Kommunikationswirkung zeigen, dass sich kommunikative Aktivitäten gegenseitig beeinflussen können (z.B. Chang/ Thorson 2004; Pfefferkorn 2009). Für den vorliegenden Kontext bedeutet dies, dass möglicherweise Wechselwirkungen zwischen den betrachteten Sponsoringarten und weiteren vom Unternehmen eingesetzten Kommunikationsinstrumenten bestehen. Diese können die Wirkung des Sponsoring auf das Markenimage stärken bzw. schwächen. In künftigen Studien ist daher eine Prüfung auf Interaktionseffekte und deren Wirkung auf das Markenimage vorzunehmen. Im vorliegenden Artikel erfolgte eine globale Messung der Sponsoringqualität. Damit bleibt unklar, wie die Sponsoringmassnahmen konkret auszugestalten sind, damit diese als positiv wahrgenommen werden. Das Wissen über die Ausgestaltung der Massnahmen wäre jedoch für die Ableitung konkreter Implikationen von grosser Bedeutung. Künftig ist daher über die Erweiterung des Modells um die Einflussfaktoren einer hohen Sponsoringqualität nachzudenken. 


\section{Literaturhinweise}

Acito, F. (1980): Employee Attitudes Toward Advertising, in: Journal of Business Research, Jg. 8, Nr. 4, S. 525-540.

Aaker, J./Fournier, S./Brasel, S. (2004): When Good Brands Do Bad, in: Journal of Consumer Research, Jg. 31, Nr. 1, S. 1-16.

Auh, S./Bell, S.J./McLeod, C.S./Shih, E. (2007): Co-production and customer loyalty in financial services, in: Journal of Retailing, Jg. 83, Nr. 3, S. 359-370.

Batt, V. (2013): Qualität der Internen Markenführung. Konzeptualisierung, empirische Befunde und Steuerung eines markenkonformen Mitarbeiterverhaltens, Wiesbaden.

Batra, R./Stephens, D. (1994): Attitudinal Effects of Ad-Evoked Moods and Emotions: The Moderating Role of Motivation, in: Psychology \& Marketing, Jg. 11, Nr. 3, S. 199-215.

Bongard, J. (2002): Werbewirkungsforschung. Grundlagen - Probleme - Ansätze, Band 7, Münster u.a.

Brammer, S./Millington, A./Rayton, B. (2007): The contribution of corporate social responsibility to organizational commitment, in: The International Journal of Human Resource Management, Jg. 18, Nr. 10, S. 1701-1719.

Brexendorf, T.O./Tomczak, T. (2004): Interne Markenführung, in: Albers, S./Tomczak, T./Hassmann, V. (Hrsg.): Verkauf - Kundenmanagement, Vertriebssteuerung, E-Commerce, Digitale Fachbibliothek, Sektion 03.15 Personal und Führung, Düsseldorf, S. 1-25.

Bruhn, M. (2004): Markenführung und Sponsoring, in: Bruhn, M. (Hrsg.): Handbuch Markenführung. Kompendium zum erfolgreichen Markenmanagement. Strategien - Instrumente - Erfahrungen, Bd. 2, 2. Aufl., Wiesbaden, S. 1593-1630.

Brubn, M. (2008): Mit Sponsoring bei den Mitarbeitenden punkten, in: io new management, o. Jg., Nr. 12, S. 8-11.

Brubn, M. (2010): Sponsoring. Systematische Planung und integrativer Einsatz, 5. Aufl., Wiesbaden.

Brubn, M./Batt, V./Köhne, Ch. (2010): Markenliebe bei Mitarbeitern - Konzeptualisierung, Determinanten und Konsequenzen auf Basis einer empirischen Untersuchung in: Marketing ZFP, Jg. 32, Nr. 2, S. 71-90.

Bryant, M.D. (2010): Commitment in Kundenbeziehungen. Eine multipartiale Messung und Analyse von Determinanten und Erfolgswirkungen, Wiesbaden.

Burke, M./Edell, J.A. (1989): The Impact of Feelings on Ad-Based Affect and Cognition, in: Journal of Marketing Research, Jg. 26, Nr.1, S. 69-83.

Burmann, C./Zeplin, S. (2005): Innengerichtetes identitätsbasiertes Markenmanagement, in: Meffert, H./Burmann, C./Koers, M. (Hrsg.): Markenmanagement. Identitätsorientierte Markenführung und praktische Umsetzung, 2. Aufl., Wiesbaden, S. 115-139.

Chen, C.-W./Shen, C.-C./Chiu, W.-U. (2007): Marketing communication strategies in support of product launch: An empirical study of Taiwanese high-tech firms, in: Industrial Marketing Management, Jg. 36, Nr. 8, S. 1046-1056.

Chang, Y./Thorson, E. (2004): Television and Web Advertising Synergies, in: Journal of Advertising, Jg. 33, Nr. 2, S. 75-84.

Cornwell, T.B./Roy, D.P./Steinard, E.A. II (2001): Exploring Managers' Perceptions of the Impact of Sponsorship on Brand Equity, in: Journal of Advertising, Jg. 30, Nr. 2, S. 41-51.

De Chernatony, L. (2002): Living the Corporate Brand: Brand Values and Brand Enactment, in: Corporate Reputation Review, Jg. 5, Nr. 2/3, S. 114-132. 
Devasagayam, P.R./Buff, C.L./Aurand, T.W./Judson, K.M. (2010): Building brand community membership within organizations: a viable internal branding alternative?, in: Journal of Product \& Brand Management, Jg. 19, Nr. 3, S. 210-217.

Drolet A./Williams, P./Lau-Gesk, L. (2007): Age-related differences in responses to affective vs. rational ads for hedonic vs. utilitarian products, in: Marketing Letters, Jg. 18, Nr. 4, S. 211-221.

Esch, F.-R./Langner, T./Schmitt, B.H./Geus, P. (2006): Are Brands Forever? How Brand Knowledge and Relationships Affect Current and Future Purchases, in: Journal of Product \& Brand Management, Jg. 15, Nr. 2, S. 98-105.

Esch, F.-R./Strödter, K. (2012): Aufbau des Markencommitment in Abhängigkeit des MitarbeiterMarken-Fits, in: Tomczak, T./Esch, F.-R./Kernstock, J./Herrmann, A. (Hrsg.): Behavioral Branding. Wie Mitarbeiterverhalten die Marke stärkt, 3. Aufl., Wiesbaden, S. 141-160.

FASPO (Fachverband Sponsoring) (2013): Sponsor Visions 2012, Hamburg.

Fornell, C./Larcker, D.A. (1981): Evaluating Structural Equation Models with Unobservable Variables and Measurement Error, in: Journal of Marketing Research, Jg. 18, Nr. 1, S. 39-50.

Giersch, J. (2008): Corporate Brand Management international tätiger Unternehmen, Wiesbaden.

Gilly, M.C./Wolfinbarger, M. (1998): Advertising's Internal Audience, in: Journal of Marketing, Jg. 62, Nr. 1, S. 69-88.

Grohs, R./Wagner, U./Vsetecka, S. (2004): Assessing the Effectiveness of Sport Sponsorships - An Empirical Examination, in: Schmalenbach Business Review (SBR), Jg. 56, Nr. 2, S. 119-138.

Gwinner, K./Bennett, G. (2008): The impact of brand cohesiveness and sport identification on brand fit in a sponsorship context, in: Journal of Sport Management, Jg. 22, Nr. 4, S. 410-426.

Harris, P. (2007): We the people: The importance of employees in the process of building customer experience, in: Journal of Brand Management, Jg. 15, Nr. 2, S. 102-114.

Hermanns, A./Kiendl, S.C./Ringle, T. (2007): Die Bedeutung des Sponsoring für die Markenführung, in: Florack, A./Scarabis, M./Primosch, E. (Hrsg.): Psychologie der Markenführung, München, S. 397-405.

Hickman, T.M./Lawrence, K.E./Ward, J.C. (2005): A Social Identities Perspective on the Effects of Corporate Sport Sponsorship on Employees, in: Sport Marketing Quarterly, Jg. 14, Nr. 3, S. 148-157.

Homburg, C./Giering, A. (1996): Konzeptualisierung und Operationalisierung komplexer Konstrukte - Ein Leitfaden für die Marketingforschung, in: Marketing ZFP, Jg. 18, Nr. 1, S. 5-24.

Homburg, C. (1998): Kundennähe von Industriegüterunternehmen, 2. Aufl., Wiesbaden.

Homburg, C./Jensen, O. (2007): The Thought Worlds of Marketing and Sales: Which Differences Make a Difference?, in: Journal of Marketing, Jg. 71, Nr. 3, S. 124-142.

Huber, F./Matthes, I. (2007): Sponsoringwirkung auf Einstellung und Kaufabsicht. Theoretische Grundlagen und Ergebnisse einer empirischen Studie, in: Marketing ZFP, 29. Jg., Nr. 2, S. 90-104.

Hughes, D.E. (2013): This ad's for you: the indirect effect of advertising perceptions on salesperson effort and performance, in: Journal of the Academy of Marketing Science, Jg. 41, Nr. 1, S. 1-18.

Jarvis, C.B./MacKenzie, S.B./Podsakoff, P.M./Mick, D.G./Bearden, W.O. (2003): A critical review of construct indicators and measurement model misspecification in marketing and consumer research, in: Journal of Consumer Research, Jg. 30, Nr. 2, S. 199-218.

Keller, K.L. (1993): Conceptualizing, Measuring, Managing Customer-Based Brand Equity, in: Journal of Marketing, Jg. 57, Nr. 1, S. 1-22. 
Keller, K.L. (2005): Kundenorientierte Messung des Markenwerts, in: Esch, F.- R. (Hrsg.): Moderne Markenführung. Grundlagen - Innovative Ansätze - Praktische Umsetzungen, 4. Aufl., Wiesbaden, S. 1307-1327.

König, V. (2010): Markenmanagement im Call Center: Eine empirische Analyse zur Konzeptionalisierung, Operationalisierung und Wirkung von Maßnahmen zum Aufbau von Brand Commitment in Call Centern, Wiesbaden.

Lardinoit, T./Quester, P.G. (2001): Attitudinal Effects of Combined Sponsorship and Sponsor's Prominence on Basketball in Europe, in: Journal of Advertising Research, Jg. 41, Nr. 1, S. 48-58.

Low, G. S./Lamb, W. L. Jr. (2000): The measurement and dimensionality of brand associations, in: Journal of Product \& Brand Management, Jg. 9, Nr. 6, S. 350-370.

Madden, T.J./Allen, C.T./Twible, J.L. (1988): Attitude Toward the Ad: An Assessment of Diverse Measurement Indices Under Different Processing 'Sets', in: Journal of Marketing Research, Jg. 25, Nr. 3, S. 242-252.

Maloney, P.B. (2007): Absatzmittlergerichtetes, identitätsbasiertes Markenmanagement, Wiesbaden.

Martensen, A./Grønholt, L./Bendtsen, L./Juul, M. (2007): Application of a model for the effectiveness of event marketing, in: Journal of Advertising Research, Jg. 47, Nr. 3, S. 283-301.

Mason, R.B./Cochetel, F. (2006): Residual Brand Awareness Following the Termination of a Longterm Event Sponsorship and the Appointment of a New Sponsor, in: Journal of Marketing Communications, Jg. 26, Nr. 2, S. 125-144.

Mazodier, M./Merunka, D. (2012): Achieving brand loyalty through sponsorship: the role of fit and self-congruity, in: Journal of the Academy of Marketing Science, Jg. 40, Nr. 6, S. 807-820.

O’Reilly III, C./Chatman, J. (1986): Organizational Commitment and Psychological Attachment: The Effects of Compliance, Identification and Internalization on Prosocial Behavior, in: Journal of Applied Psychology, Jg. 71, Nr. 3, S. 492-499.

Park, J.W./Kim K.H./Kim J.K. (2002): Acceptance of Brand Extensions: Interactive Influences of Product Category Similarity, Typicality of Claimed Benefits and Brand Relationship Quality, in: Advances in Consumer Research, Jg. 29, Nr. 1, S. 190-198.

Panagopoulos, N.G./Rapp, A./Vlachos, P.A. (2011): Corporate Social Performance and Employees: Construed Perceptions, Attributions and Behavioral Outcomes, Working Paper Series.

Pfefferkorn, E.J. (2009): Kommunikationscontrolling in Verbindung mit Zielgrößen des Markenwerts. Eine methodische Herangehensweise und Prüfung an einem Fallbeispiel, Wiesbaden.

Piehler, R. (2011): Interne Markenführung. Theoretisches Konzept und fallstudienbasierte Evidenz, Wiesbaden.

Rowley, J./Williams, C. (2008): The impact of brand sponsorship of music festivals, in: Marketing Intelligence \& Planning, Jg. 26, Nr. 7, S. 781-792.

Tomczak, T./Reinecke, S./Dittrich, S. (2008): Kundenbindung durch Kundenkarten und -clubs, in Brubn, M./Homburg, C. (Hrsg.): Handbuch Kundenbindungsmanagement. Strategien und Instrumente für ein erfolgreiches CRM, 6. Aufl., Wiesbaden, S. 323-345.

Van Dyne, L./Graham, J.W./Dienesch, R.M. (1994): Organizational Citizenship Behavior: Construct Redefinition, Measurement and Validation, in: Academy of Management Journal, Jg. 37, Nr. 4, S. 765-802.

Wentzel, D./Henkel, S./Tomczak, T. (2009): Die Rolle der Werbung in der internen Markenführung, in: Marketing ZFP, Jg. 31, Nr. 1, S. 43-56. 
Wentzel, D./Henkel, S./Tomczak, T. (2010): Can I Live Up to That Ad? Impact of Implicit Theories of Ability on Service Employees' Responses to Advertising, in: Journal of Service Research, Jg. 13, Nr. 2, S. 137-152.

Wolfinbarger, M.F./Gilly, M.C. (2005): How Firm Advertising Affects Employees‘ Trust, Organizational Identification, and Customer Focus, in: MSI Reports, Nr. 05-002, S. 21-39.

Wolfinbarger Celsi, M./Gilly, M.C. (2010): Employees as internal audience: how advertising affects employees' customer focus, in: Journal of the Academy of Marketing Science, Jg. 38, Nr. 4, S. 520-529.

Zeplin, S. (2006): Innengerichtetes identitätsbasiertes Markenmanagement, Wiesbaden. 


\section{Anhang}

\begin{tabular}{|c|c|c|}
\hline \multicolumn{3}{|l|}{ Sportsponsoringqualität } \\
\hline Itemformulierung & $\begin{array}{l}\text { Standardisierte } \\
\text { Faktorladung } \\
\text { (KFA) }\end{array}$ & Quelle \\
\hline $\begin{array}{l}\text { Das Engagement der Marke XX im Be- } \\
\text { reich Sport finde ich gut. }\end{array}$ & $0.83 * * *$ & \multirow{3}{*}{$\begin{array}{l}\text { In Anlehnung an Chen et al. } \\
\text { 2007; } \\
\text { Drolet et al. } 2007\end{array}$} \\
\hline $\begin{array}{l}\text { Das Engagement der Marke XX im Be- } \\
\text { reich Sport entspricht meinen Erwartun- } \\
\text { gen. }\end{array}$ & $0.78 * * *$ & \\
\hline $\begin{array}{l}\text { Das Engagement der Marke XX im Be- } \\
\text { reich Sport spricht mich an. }\end{array}$ & $0.83 * * *$ & \\
\hline \multicolumn{3}{|c|}{$\alpha=0.85 ;$ Konstruktreliabilität $=0.85 ;$ Varianzerklärung $=77.3 \%$} \\
\hline \multicolumn{3}{|l|}{ Kultursponsoringqualität } \\
\hline Itemformulierung & $\begin{array}{l}\text { Standardisierte } \\
\text { Faktorladung } \\
\text { (KFA) }\end{array}$ & Quelle \\
\hline $\begin{array}{l}\text { Das Engagement der Marke XX im Be- } \\
\text { reich Kultur finde ich gut. }\end{array}$ & $0.84 * *$ & \multirow{3}{*}{$\begin{array}{l}\text { In Anlehnung an Chen et al. } \\
\text { 2007; } \\
\text { Drolet et al. } 2007\end{array}$} \\
\hline $\begin{array}{l}\text { Das Engagement der Marke XX im Be- } \\
\text { reich Kultur entspricht meinen Erwar- } \\
\text { tungen. }\end{array}$ & $0.79 * * *$ & \\
\hline $\begin{array}{l}\text { Das Engagement der Marke XX im Be- } \\
\text { reich Kultur spricht mich an. }\end{array}$ & $0.88 * * *$ & \\
\hline \multicolumn{3}{|c|}{$\alpha=0.87 ;$ Konstruktreliabilität $=0.88 ;$ Varianzerklärung $=80.0 \%$} \\
\hline \multicolumn{3}{|c|}{ Soziosponsoringqualität } \\
\hline Itemformulierung & $\begin{array}{l}\text { Standardisierte } \\
\text { Faktorladung } \\
\text { (KFA) }\end{array}$ & Quelle \\
\hline $\begin{array}{l}\text { Das Engagement der Marke XX im so- } \\
\text { zialen Bereich finde ich gut. }\end{array}$ & $0.78 * * *$ & \multirow{3}{*}{$\begin{array}{l}\text { In Anlehnung an Chen et al. } \\
\text { 2007; } \\
\text { Drolet et al. } 2007\end{array}$} \\
\hline $\begin{array}{l}\text { Das Engagement der Marke XX im so- } \\
\text { zialen Bereich entspricht meinen Erwar- } \\
\text { tungen. }\end{array}$ & $0.64 * * *$ & \\
\hline $\begin{array}{l}\text { Das Engagement der Marke XX im so- } \\
\text { zialen Bereich spricht mich an. }\end{array}$ & $0.83 * *$ & \\
\hline \multicolumn{3}{|c|}{$\alpha=0.78 ;$ Konstruktreliabilität $=0.79 ;$ Varianzerklärung $=70.2 \%$} \\
\hline
\end{tabular}




\begin{tabular}{|c|c|c|}
\hline \multicolumn{3}{|l|}{ Umweltsponsoringqualität } \\
\hline Itemformulierung & $\begin{array}{l}\text { Standardisierte } \\
\text { Faktorladung } \\
\text { (KFA) }\end{array}$ & Quelle \\
\hline $\begin{array}{l}\text { Das Engagement der Marke XX im Be- } \\
\text { reich Umwelt finde ich gut. }\end{array}$ & $0.81 * * *$ & \multirow{3}{*}{$\begin{array}{l}\text { In Anlehnung an Chen et al. } \\
\text { 2007; } \\
\text { Drolet et al. } 2007\end{array}$} \\
\hline $\begin{array}{l}\text { Das Engagement der Marke XX im Be- } \\
\text { reich Umwelt entspricht meinen Erwar- } \\
\text { tungen. }\end{array}$ & $0.80 * * *$ & \\
\hline $\begin{array}{l}\text { Das Engagement der Marke XX im Be- } \\
\text { reich Umwelt spricht mich an. }\end{array}$ & $0.86 * * *$ & \\
\hline \multicolumn{3}{|c|}{$\alpha=0.86 ;$ Konstruktreliabilität $=0.86 ;$ Varianzerklärung $=78.6 \%$} \\
\hline \multicolumn{3}{|c|}{ Markenimage } \\
\hline Itemformulierung & $\begin{array}{l}\text { Standardisierte } \\
\text { Faktorladung } \\
\text { (KFA) }\end{array}$ & Quelle \\
\hline Ich erlebe die Marke XX als dynamisch. & $0.80 * *$ & \multirow{4}{*}{$\begin{array}{l}\text { In Anlehnung an Low/Lamb } \\
2000 ; \text { Esch et al. 2006; Keller } \\
2005\end{array}$} \\
\hline Ich erlebe die Marke XX als begeisternd. & $0.84 * * *$ & \\
\hline Ich erlebe die Marke XX als nachhaltig. & $0.71 * * *$ & \\
\hline $\begin{array}{l}\text { Ich erlebe die Marke XX als nahe beim } \\
\text { Kunden. }\end{array}$ & $0.81 * * *$ & \\
\hline \multicolumn{3}{|c|}{$\alpha=0.87 ;$ Konstruktreliabilität $=0.92 ;$ Varianzerklärung $=71.7 \%$} \\
\hline \multicolumn{3}{|c|}{ Markencommitment } \\
\hline Itemformulierung & $\begin{array}{l}\text { Standardisierte } \\
\text { Faktorladung } \\
(\text { KFA })\end{array}$ & Quelle \\
\hline $\begin{array}{l}\text { Wenn ich über die Marke XX rede, spre- } \\
\text { che ich von „wir“ bzw. „uns“. }\end{array}$ & $0.56 * * *$ & Aaker et al. 2004 \\
\hline $\begin{array}{l}\text { Ich fühle mich der Marke XX sehr ver- } \\
\text { bunden. }\end{array}$ & $0.91 * * *$ & Park et al. 2002 \\
\hline $\begin{array}{l}\text { Ich erzähle meinen Freunden oder Be- } \\
\text { kannten gerne, dass ich für die Marke } \\
\text { XX arbeite. }\end{array}$ & $0.71 * * *$ & Brubn et al. 2010; Batt 2013 \\
\hline \multicolumn{3}{|c|}{$\alpha=0.72 ;$ Konstruktreliabilität $=0.78 ;$ Varianzerklärung $=67.7 \%$} \\
\hline
\end{tabular}




\begin{tabular}{|c|c|c|}
\hline \multicolumn{3}{|c|}{ Markenkonformes Mitarbeiterverhalten } \\
\hline Konstruktdimension & $\begin{array}{l}\text { Standardisierte } \\
\text { Faktorladung } \\
\text { (KFA) }\end{array}$ & Quelle \\
\hline Markenakzeptanz (Index) & $0.69 * * *$ & $\begin{array}{l}\text { In Anlehnung an Maloney } \\
\text { 2007; König 2010; Piebler } \\
2011\end{array}$ \\
\hline Markenmissionierung (Index) & $0.91 * * *$ & $\begin{array}{l}\text { In Anlehnung an Van Dyne et } \\
\text { al. 1994; Maloney 2007; Kö- } \\
\text { nig 2010; Piehler } 2011\end{array}$ \\
\hline Markenpartizipation (Index) & $0.65 * * *$ & $\begin{array}{l}\text { In Anlehnung an Zeplin } \\
\text { 2006; Maloney 2007; Piebler } \\
2011\end{array}$ \\
\hline \multicolumn{3}{|c|}{$\alpha=0.80 ;$ Konstruktreliabilität $=0.80 ;$ Varianzerklärung $=71.5 \%$} \\
\hline \multicolumn{3}{|c|}{$\begin{array}{l}\chi^{2}=477.44 ; \mathrm{df}=188.00 ; \chi 2 / \mathrm{df}=2.54 ; \mathrm{NFI}=0.84 ; \mathrm{TLI}=0.87 ; \mathrm{CFI}=0.90 ; \mathrm{SRMR}=0.06 ; \mathrm{RMSEA}= \\
0.08 \\
\text { Legende: } \quad * * \text { signifikant auf } 0.1 \%-\text { Niveau }\end{array}$} \\
\hline
\end{tabular}

Tabelle A1: Operationalisierung und Validierung der Variablen des Messmodells

\begin{tabular}{|c|c|c|c|c|c|c|c|}
\hline & UmwS & SozS & KultS & SpoS & IMG & $\mathrm{COM}$ & MKMV \\
\hline UmwS & 0.82 & & & & & & \\
\hline SozS & 0.50 & 0.75 & & & & & \\
\hline KultS & 0.46 & 0.55 & 0.84 & & & & \\
\hline SpoS & 0.29 & 0.34 & 0.53 & 0.81 & & & \\
\hline IMG & 0.48 & 0.43 & 0.63 & 0.57 & 0.87 & & \\
\hline $\mathrm{COM}$ & 0.25 & 0.23 & 0.33 & 0.30 & 0.52 & 0.74 & \\
\hline MKMV & 0.19 & 0.17 & 0.25 & 0.22 & 0.39 & 0.74 & 0.76 \\
\hline Legende: & \multicolumn{7}{|c|}{$\begin{array}{l}\text { In der Diagonalen (gefettet) stehen die Wurzeln der DEV-Werte, unterhalb der Diagonalen } \\
\text { die Faktorkorrelationen. } \\
\text { DEV = Durchschnittlich erfasste Varianz, UmwS = Umweltsponsoring, SozS = Soziosponso- } \\
\text { ring, KultS = Kultursponsoring, SpoS = Sportsponsoring, IMG = Image, Com = Commit } \\
\text { ment, MKMV = Markenkonformes Mitarbeiterverhalten }\end{array}$} \\
\hline
\end{tabular}

Tabelle A2: Prüfung der Modellkonstrukte auf Diskriminanzvalidität 
Manfred Bruhn, Prof. Dr., ist Inhaber des Lehrstuhls für Marketing und Unternehmensführung an der Wirtschaftswissenschaftlichen Fakultät der Universität Basel und Honorarprofessor an der Technischen Universität München.

Verena Batt, Dr. rer. pol., ist wissenschaftliche Mitarbeiterin am Lehrstuhl für Marketing und Unternehmensführung an der Wirtschaftswissenschaftlichen Fakultät der Universität Basel.

Matthias Holzer, Dipl.-Kfm. techn., EMBS ist wissenschaftlicher Mitarbeiter am Lehrstuhl für Marketing und Unternehmensführung an der Wirtschaftswissenschaftlichen Fakultät der Universität Basel.

Anschrift: Universität Basel, Wirtschaftswissenschaftliche Fakultät, Lehrstuhl für Marketing und Unternehmensführung, Peter Merian-Weg 6, CH-4002 Basel, Tel.: +41 61267 32 22, Fax: +41 6126728 38, E-Mail: manfred.bruhn@unibas.ch, verena.batt@unibas.ch, m.holzer@unibas.ch

Wir danken Herrn Cédric Pezzali (Master of Science in Business and Economics) für sein Engagement bei der Erhebung der empirischen Daten. 